\title{
Metabolic PET/CT-Guided Lung Lesion Biopsies: Impact on Diagnostic Accuracy and Rate of Sampling Error
}

\author{
Ludmila Guralnik*1, Radu Rozenberg*1, Alex Frenkel², Ora Israel ${ }^{2,3}$, and Zohar Keidar ${ }^{2,3}$ \\ ${ }^{I}$ Department of Radiology, Rambam Health Care Campus, Haifa, Israel; ${ }^{2}$ Department of Nuclear Medicine, Rambam Health Care \\ Campus, Haifa, Israel; and ${ }^{3} B \& R$ Rappaport Faculty of Medicine, Technion-Israel Institute of Technology, Haifa, Israel
}

CT-guided fine-needle aspiration (FNA) of lung lesions is subject to sampling errors. The current study assessed whether information provided by ${ }^{18} \mathrm{~F}-\mathrm{FDG}$ PET/CT will decrease the false-negative (FN) rate and thus improve the accuracy of CT-guided FNA. Methods: Data from 311 consecutive patients with lung nodules who underwent ${ }^{18} \mathrm{~F}$-FDG PET/CT and CT-guided FNA within an interval of less than $30 \mathrm{~d}$ were retrospectively assessed. In-house-developed software was used to register CT images performed for the FNA procedure (CT FNA) with corresponding slices of the PET/CT study. The quality of registration was rated on a scale of 1 (excellent) to 5 (misregistration). Only cases scored 1 or 2 were further evaluated. The software provided the highest standardized uptake value (SUV) within the lesion and at the location of the tip of the aspirating needle. The distance between the tip and the area with the highest SUV within the lesion was measured. The mean distance from the tip of the needle to the focus with the highest SUV, as well as the mean difference between the maximum SUV in the whole lesion and at the needle tip, was calculated and compared for cases with truepositive (TP) and FN FNA results. Anatomic and metabolic parameters of lesions included in these 2 groups were also compared. Results: There were 267 patients (86\%) with score 1 or 2 registration quality for CT FNA and PET/CT/CT images, including 179 TP (67\%), 5 false-positive (FP, 2\%), 49 true-negative (TN, 18\%), and 34 FN (13\%) FNA results. The distance between the location of the needle tip and the focus with the highest SUV in the lesion was significantly greater in the FN group $(15.4 \pm 14 \mathrm{~mm})$ than in the TP group $(5.9 \pm 13.4 \mathrm{~mm}, P<0.001)$. The maximum SUV at the location of the aspirating needle tip was significantly higher in the TP group, at $6.4 \pm 6.4$, than in the FN group, at $4 \pm 4.7(P<0.05)$. Conclusion: The present results demonstrate a relationship between the degree of metabolism at the site of tissue-sampling aspiration in lung lesions and the accuracy of FNA results. Anatomy- and metabolism-based FNA guidance using information provided by both ${ }^{18} \mathrm{~F}-\mathrm{FDG}$ PET and CT may improve the accuracy of histologic examinations, decrease the rate of $\mathrm{FN}$ results, and thus increase the probability of achieving a definitive diagnosis.

Key Words: biopsy; PET/CT; lung; CT-guided biopsy

J Nucl Med 2015; 56:518-522

DOI: 10.2967/jnumed.113.131466

Received Nov. 18, 2014; revision accepted Jan. 13, 2015.

For correspondence or reprints contact: Zohar Keidar, Department of Nuclear Medicine, Rambam Health Care Campus, Bat-Galim, Haifa 35254, Israel.

E-mail: zohar@keidar.net

${ }^{*}$ Contributed equally to this work.

Published online Feb. 19, 2015.

COPYRIGHT (c) 2015 by the Society of Nuclear Medicine and Molecular Imaging, Inc.
$\mathbf{P}$ ercutaneous CT-guided fine-needle aspiration (FNA) is routinely used for lung lesions and is a relatively safe method for diagnosis of benign and malignant processes (1). The diagnostic accuracy of CT-guided FNA for malignant lung tumors varies between $64 \%$ and $97 \%$ (2), depending on factors such as the size and depth of the lesion and the number of needle paths $(1,2) .{ }^{18} \mathrm{~F}$ FDG imaging provides information on the metabolic characteristics of lung lesions (3). Its inherent advantages include early detection of malignancy and differentiation from nonmalignant areas within a mass representing fibrosis and necrotic tissue (3-5). ${ }^{18}$ F-FDG PET/CT has a high sensitivity of approximately $90 \%$ in characterizing solitary pulmonary nodules, but its specificity is hampered by ${ }^{18} \mathrm{~F}$-FDG accumulation by various nonmalignant processes $(6,7)$. CT used for guidance of FNA does not provide information on the metabolic characteristics of the lesion or, particularly, the specific location of functionally active cells within the tumor (8). Tissue aspiration guided only by anatomic data can therefore lead to placement of the needle into a nonviable area of the lesion with subsequent inadequate tissue sampling and potentially false-negative (FN) FNA results $(8,9)$.

The success rate of image-guided biopsies might be improved and multiple attempts at biopsy avoided if the functional information obtained from the ${ }^{18} \mathrm{~F}$-FDG PET/CT study is used to direct the positioning of the tip of the needle to the region with the highest metabolism within a lesion (9). The purpose of this study was to demonstrate that ${ }^{18} \mathrm{~F}$-FDG PET/CT can provide the additional information that will decrease the FN rate and improve the accuracy of CT-guided FNA of lung lesions.

\section{MATERIALS AND METHODS}

\section{Study Population}

The institutional ${ }^{18} \mathrm{~F}-\mathrm{FDG}$ PET/CT and CT-guided biopsy databases for 2007 to 2010 were retrospectively searched for patients who, within an interval of less than $30 \mathrm{~d}$, underwent whole-body FDG PET/CT for characterization of pulmonary nodules and CT-guided FNA of the lung lesion. Medical record data for 311 consecutive patients were retrieved, including the cytology results of samples obtained at CT-guided FNA and histology of the same lesions obtained at surgery, when performed, as well as clinical and imaging follow-up. The institutional review board approved this retrospective study, and the requirement to obtain informed consent was waived.

\section{CT-Guided FNA Procedure}

CT-guided FNA was performed as an unenhanced CT procedure (MX 8000, 4 slices, or IDT, 16 slices; Philips) by senior radiologists with experience ranging between 9 and 20 y. A 22-gauge needle, 9 or $15 \mathrm{~cm}$ in length, was used. A cytologist evaluated the initial aspiration 
and prepared the slides. Cytology results were categorized as positive if malignant cells were identified, benign if a specific nononcologic diagnosis could be made, and negative if only respiratory cells or macrophages were present. FNA results were considered truepositive (TP) if malignant cells were detected and further confirmed at surgery or by imaging and clinical follow-up and true-negative (TN) if no evidence of malignancy was found on cytology and the patients had either a benign lesion at surgery or no evidence of cancer during clinical follow-up to $5 \mathrm{y}$. In false-positive (FP) cases, the presence of malignant cells was reported on FNA results with no further evidence of malignancy, whereas in FN cases no malignant cells were found on aspiration but cancer was diagnosed in that lesion at surgery.

\section{PET/CT Acquisition and Processing}

Patients were instructed to fast, except for glucose-free oral hydration, for 4-6 $\mathrm{h}$ before the injection of $370-555 \mathrm{MBq}(10-15 \mathrm{mCi})$ of ${ }^{18}$ F-FDG. Diabetic patients were instructed to keep to their regular glucose-controlling drug schedule. Blood glucose levels were measured before injection. PET and unenhanced CT were performed consecutively 60-90 min after the injection of ${ }^{18} \mathrm{~F}-\mathrm{FDG}$, using a PET/CT system (Discovery LS or Discovery 690; GE Healthcare). The patients were instructed to breath normally during the PET/CT acquisition. For the purpose of this research, all studies were reviewed retrospectively with knowledge of the patient's clinical history and results of previous imaging tests, masked to the results of the FNA examination. ${ }^{18} \mathrm{~F}-\mathrm{FDG}$ activity in the lung lesions was graded as homogeneous, inhomogeneous, or absent. Each lung lesion was assessed on CT images for size and pattern as solid, semisolid, or ground glass opacity.

\section{Registration Between PET/CT and CT-Guided FNA Data}

In-house-developed software was used to register CT images performed for the FNA procedure (CT FNA) with corresponding slices of the PET/CT study. The software was implemented on the Xeleris workstation (GE Healthcare). The registration was performed by a radiologist and took less than 1 min per patient using the last slice of the CT FNA series. In this image, the location of the needle tip within the lung lesion represented the site of aspiration. The operator then manually scrolled through the CT slices of the PET/CT study to identify the image in which the contours of the CT FNA matched those of the PET/CT image. The quality of registration was rated visually by the operator on a scale of 1-5 as 1 (excellent) if the registration contours matched the lesion, lungs, and mediastinum; 2 (good) if the registration contours matched the lesion with no accurate match of the lungs and mediastinum; 3 (fair) if the registration contours included the lesion but with no accurate match of its perimeter; 4 (poor) if the registration contours included the lesion but with a significantly inaccurate match; or 5 if no registration could be obtained. Only cases scored 1 or 2 were further evaluated and included in the final data analysis. After registration, a matched CT FNA and ${ }^{18}$ F-FDG PET image was available for each patient. The software further provided measurements of the maximum standardized uptake value $\left(\mathrm{SUV}_{\max }\right)$ in a $3 \times 3$ pixel cube around the location of the tip of the aspirating needle, $\mathrm{SUV}_{\max }$ of the whole lesion, and distance between the location of the tip of the needle and that of the highest ${ }^{18} \mathrm{~F}-\mathrm{FDG}$ uptake within the lesion.

\section{Statistical Analysis}

Data are presented as mean, minimum, and maximum values and $\mathrm{SD}$. Lesion size, $\mathrm{SUV}_{\max }$, and the mean distance between the location of the highest $\mathrm{SUV}_{\max }$ in the lesion and the location in which the tissue sample was obtained were measured and compared between the group of patients with TP and FN results of FNA using the MannWhitney nonparametric test with a $P$ value of less than 0.05 being considered statistically significant. Lesion size and ${ }^{18}$ F-FDG uptake patterns were compared between the TP and FN groups using the $\chi^{2}$ test.

\section{RESULTS}

Of the 311 patients who met the inclusion criteria, 185 were men and 126 women, with an age range of 19-95 y (average, $67 \mathrm{y})$. Ninety patients $(29 \%)$ had lesions in the right upper lobe, $83(27 \%)$ in the left upper lobe, $75(24 \%)$ in the right lower lobe, $53(17 \%)$ in the left lower lobe, and $10(3 \%)$ in the right middle lobe. The largest diameter of the lung lesions ranged from 6.5 to $148.6 \mathrm{~mm}$ (average, $32 \mathrm{~mm}$ ). On CT, 229 lesions (74\%) demonstrated a solid pattern, $72(23 \%)$ were semisolid, and 10 (3\%) had a ground-glass appearance. On PET, 113 lesions (36\%) demonstrated homogeneous ${ }^{18} \mathrm{~F}-\mathrm{FDG}$ uptake, 160 (51\%) were

TABLE 1

Morphologic and Metabolic Lesion Characteristics in Cases with TP and FN FNA Results

\begin{tabular}{|c|c|c|c|}
\hline Characteristic & TP & $\mathrm{FN}$ & $P$ \\
\hline No. of patients & 179 & 34 & \\
\hline Size of lesion $(\mathrm{mm})$ & & & NS \\
\hline Average & 35.2 & 29.8 & \\
\hline Minimum & 6.5 & 9 & \\
\hline Maximum & 100 & 148 & \\
\hline PET uptake pattern (\%) & & & NS \\
\hline Homogeneous & $70(39 \%)$ & $16(47 \%)$ & \\
\hline Inhomogeneous & $102(57 \%)$ & $14(41 \%)$ & NS \\
\hline Absent & $7(4 \%)$ & $4(12 \%)$ & NS \\
\hline $\mathrm{SUV}_{\max }$ at needle tip & & & $<0.05$ \\
\hline Average & 6.4 & 4 & \\
\hline Minimum & 0.3 & 0.22 & \\
\hline Maximum & 44.7 & 22.1 & \\
\hline Lesion $\mathrm{SUV}_{\max }$ & & & $<0.05$ \\
\hline Average & 12.4 & 9.3 & \\
\hline Minimum & 0.84 & 0.3 & \\
\hline Maximum & 63.7 & 29.8 & \\
\hline $\begin{array}{l}\text { Distance from needle tip } \\
\text { to highest tumor } \\
\mathrm{SUV}_{\max }(\mathrm{mm})\end{array}$ & & & $<0.001$ \\
\hline Average & 5.9 & 15.4 & \\
\hline Minimum & 0 & 0 & \\
\hline Maximum & 86 & 50.3 & \\
\hline Adenocarcinoma & $102(56 \%)$ & $13(38 \%)$ & \\
\hline $\begin{array}{r}\text { Squamous cell } \\
\text { carcinoma }\end{array}$ & $24(13 \%)$ & $5(15 \%)$ & \\
\hline Small cell lung cancer & $3(2 \%)$ & $1(3 \%)$ & \\
\hline $\begin{array}{l}\text { Non-small cell lung } \\
\text { cancer* }\end{array}$ & $28(15 \%)$ & $1(3 \%)$ & \\
\hline Neuroendocrine tumor & $4(2 \%)$ & $7(20.5 \%)$ & \\
\hline Other malignancies $^{\dagger}$ & $21(12 \%)$ & $7(20.5 \%)$ & \\
\hline
\end{tabular}

*No surgery was performed.

${ }^{\dagger}$ Metastases, lymphomas, and nonspecific malignant cells. 


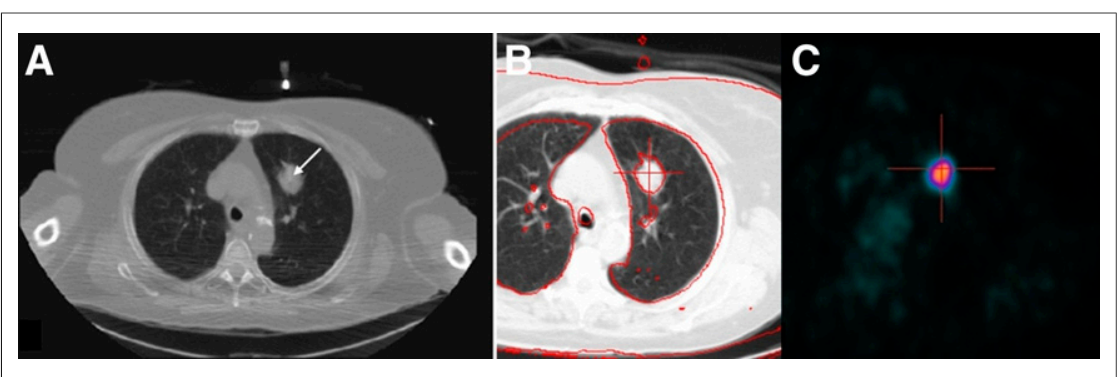

FIGURE 1. TP CT-guided FNA in $44 \times 42 \mathrm{~mm}$ lesion in left lung: CT FNA demonstrating location of aspirating needle (arrow) (A), registered CT FNA slice with corresponding PET/CT image (red contours, score 1) (B), and corresponding ${ }^{18} \mathrm{~F}-\mathrm{FDG}$ PET slice demonstrating high and homogeneous ${ }^{18} \mathrm{~F}-\mathrm{FDG}$ uptake at site of needle tip (C). SUV $\max$ at tip of needle: 9.1 ; highest SUV $_{\max }$ in tumor: 11.0, distance between tumor SUV $_{\max }$ and tip of needle: $3.77 \mathrm{~mm}$; FNA results: adenocarcinoma; histologic sample obtained at surgery: poorly differentiated adenocarcinoma.

inhomogeneous, and $38(12 \%)$ showed no abnormal ${ }^{18} \mathrm{~F}-\mathrm{FDG}$ uptake. CT FNA positioning was prone in 155 patients $(50 \%)$, supine in $113(36 \%)$, left decubitus in $23(7 \%)$, and right decubitus in $20(6 \%)$.

The registration score of CT FNA and PET/CT images was 1 in 129 patients (41\%), 2 in $138(44 \%), 3$ in $23(7 \%), 4$ in $19(6 \%)$, and 5 in $2(0.64 \%)$. A total of 267 patients $(86 \%)$ had score 1 or 2 registration quality and were further analyzed.

The FNA results were consistent with cancer in 184 patients (69\%) and showed no malignant cells or were equivocal in 83 patients (31\%). Of the 184 positive FNA results, 179 were further confirmed as malignancy on biopsy obtained at surgery or based on follow-up imaging and clinical assessment (TP, 67\%) whereas 5 were diagnosed as benign processes (FP, 2\%). Forty-nine negative FNA results were further confirmed as benign processes at surgery or on clinical follow-up (TN, 18\%), whereas 34 lesions were malignant (FN, 13\%). The sensitivity, specificity, and accuracy of FNA for diagnosis of pulmonary lesions were $84 \%, 91 \%$, and $85 \%$, respectively.

The distance between the location of the tip of the needle and the focus with the highest $\mathrm{SUV}_{\max }$ within the lesion was significantly greater in patients with FN results on FNA $(15.4 \pm 14 \mathrm{~mm})$ than in those with TP results $(5.9 \pm 13.4 \mathrm{~mm}, P<0.001)$. The $\mathrm{SUV}_{\max }$ in the tumor and the $\mathrm{SUV}_{\max }$ in the location of the aspirating needle tip were significantly higher in the TP group $(12.4 \pm 10$ and $6.4 \pm 6.4$, respectively) than in the FN group $(9.2 \pm 7.3[P<0.05]$

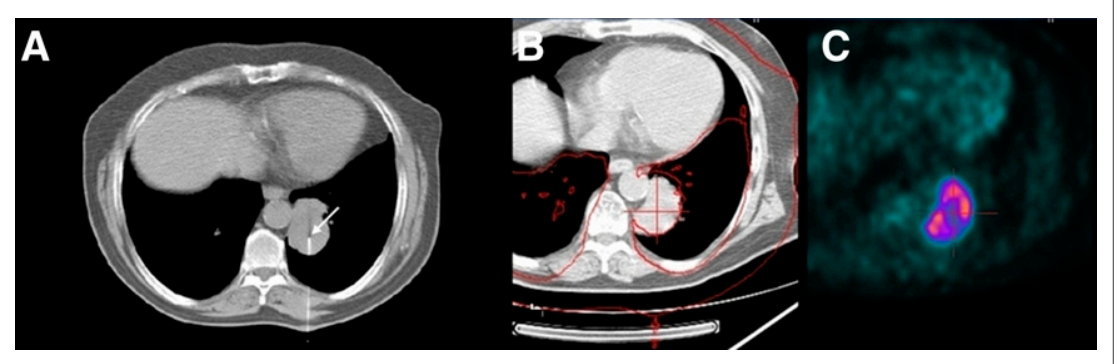

FIGURE 2. FN CT-guided FNA in $58 \times 37 \mathrm{~mm}$ lesion in left lung: CT FNA demonstrating location of aspirating needle (arrow) (A), registered CT FNA slice with corresponding PET/CT image (red contours, score 2) (B), and corresponding ${ }^{18} \mathrm{~F}-\mathrm{FDG}$ PET slice demonstrating that tip of needle is inside area of lesion with low metabolic activity (C). SUV max $_{\text {ax }}$ at tip of needle: 2.4; highest SUV max $_{\text {in }}$ tumor: 6.9; distance between tumor SUV $\mathrm{max}_{\max }$ and tip of needle: $11.85 \mathrm{~mm}$; FNA results: macrophages and respiratory cells; histologic sample obtained at surgery: moderately differentiated squamous cell carcinoma. and $4 \pm 4.7[P<0.05]$, respectively). There was no statistically significant difference in lesion size and ${ }^{18}$ F-FDG uptake pattern between patients with TP and FN results on FNA (Table 1; Figs. 1 and 2).

\section{DISCUSSION}

CT-guided FNA and ${ }^{18}$ F-FDG PET/CT examinations are performed routinely in patients with pulmonary lesions, both for diagnosis and for staging. It is critical that these tests be accurate to ensure selection of the most appropriate management plan for an individual patient. Tissue sampling of the area in which malignancy is more strongly suspected is important to ensure accurate FNA results. However, the presence and number of malignant cells may vary across or between lung masses. A larger lesion may contain malignant, highly active areas mixed with necrotic or fibrotic regions characterized, as a rule, by low or absent metabolic activity (9). Similarly, in patients with multiple lung lesions, the lesions may have varying levels of metabolic activity and their random sampling may not provide the most appropriate tissue for correct diagnosis, resulting in FN FNA tests.

${ }^{18}$ F-FDG PET/CT can detect and correctly localize foci of high metabolic activity within a lung lesion and thus distinguish viable, mainly malignant, tissue from nonmalignant tissue, thereby reducing the sampling error and increasing the diagnostic accuracy of image-guided biopsy. Alternatively, in patients with multiple lesions, PET/CT can be used to target the most metabolically active lesions, with the highest ${ }^{18}$ F-FDG uptake, for biopsy.

The results of FNA in the present study population, based only on CT-guided aspiration, showed sensitivity, specificity, and accuracy of $84 \%, 91 \%$, and $85 \%$, respectively, for diagnosis of pulmonary malignancy, similar to previously reported literature data $(2,10)$. However, this study aimed to examine whether combining CT-guided FNA and ${ }^{18} \mathrm{~F}-\mathrm{FDG}$ PET/CT data had an added value in improving the tissue-sampling accuracy. The present results demonstrate that the distance between the aspirating needle tip and the area with the highest metabolic activity within the lesion was significantly greater in patients with FN FNA results than in those with TP FNA results. In addition, $\mathrm{SUV}_{\max }$ in the location of the aspirating needle tip was significantly higher in the TP group than in the FN group. These results demonstrate the relationship between the degree of metabolism at the site of aspiration within lung lesions and the accuracy of FNA results. Interestingly, there were no significant differences in lesion size or tissue heterogeneity between the TP and FN groups. This finding is in contrast to the accepted assumptions that the larger and more heterogeneous the lesion is, the higher is the probability of sampling error, and that a lower FNA accuracy is expected in small lesions, mainly because 
of the partial-volume effect $(11,12)$. This lack of heterogeneity differences between the TP and FN groups further emphasizes the need for quantitative measurements as a tool for more accurate tissue sampling in addition to visual analysis of the assessed lesion.

Previous studies have examined the value of PET-guided biopsy in malignant lesions in the bone. In a retrospective study, ${ }^{18} \mathrm{~F}-\mathrm{FDG}$ PET-directed skeletal biopsies resulted in a higher number of TP and a lower number of FP biopsies than did sampling directed by bone scintigraphy (13). In a case report of a patient with medullary and trabecular bone metastases, only the use of ${ }^{18} \mathrm{~F}-\mathrm{FDG}$ PET/CT allowed the biopsy to be localized to an area that produced a conclusive tissue specimen containing viable tumor; CT, MR imaging, and bone scintigraphy failed to reliably detect bone marrow infiltration (14).

The present study provided support for the use of ${ }^{18} \mathrm{~F}-\mathrm{FDG}$ PET/CT to improve the diagnostic accuracy of FNA in lung lesions. In addition to guiding the operator to the most metabolically active area of a neoplasm, ${ }^{18} \mathrm{~F}$-FDG PET/CT has the advantage of guiding FNA. The use of PET/CT allows the most easily accessible lesion to be identified, thereby reducing the potential need for repeated invasive procedures and the risk of complications. PET/CT can assist in guiding the operator to a hard-to-locate metabolically active region in a lesion, such as may be the case when there are anatomic alterations after surgery or radiation therapy, or when lesions have only minimal morphologic changes on diagnostic CT. Because ${ }^{18} \mathrm{~F}-\mathrm{FDG}$ $\mathrm{PET} / \mathrm{CT}$ can detect malignancy before significant morphologic changes occur, the use of this test to guide biopsy could also help provide an earlier histologic diagnosis of small-tumorload malignancies.

Although ${ }^{18} \mathrm{~F}-\mathrm{FDG}$ PET/CT is used for diagnosis and staging of lung malignances, some metabolically active benign processes can also show high ${ }^{18}$ F-FDG uptake $(7,15-17)$. Differentiating inflammatory and infectious processes in benign tumors from malignancy may be difficult, but confidence that the aspirating needle has been placed in the right location is important in these cases as well and is higher if FNA is guided by ${ }^{18} \mathrm{~F}-\mathrm{FDG}$ imaging. Although there is increasing evidence that ${ }^{18} \mathrm{~F}-\mathrm{FDG}$ PET/CT has a role in guiding biopsies, the logistics of integrating this imaging modality into routine clinical FNA procedures is more complex. Recent reports have presented potential solutions. Previously acquired ${ }^{18} \mathrm{~F}$-FDG PET/CT images have been digitally registered with the intraprocedural $\mathrm{CT}$ images to guide needle placement during biopsy of abdominal masses (18). An additional study used coregistered, previously acquired ${ }^{18} \mathrm{~F}-\mathrm{FDG}$ PET/CT images with intraprocedural CT images and reported that in approximately $50 \%$ of biopsies on various organs, the metabolic information improved the diagnostic accuracy over CT-only guided biopsy (9). Registration of separately performed PET/CT and procedural CT data presents a few limitations. In the present large series, suboptimal registration of ${ }^{18} \mathrm{~F}-\mathrm{FDG}$ PET/CT and CT FNA was found in 14\% of cases and was related to changes in the size, shape, and metabolic characteristics of the lesion that occur during the interval between the PET/CT and the biopsy procedure. Additional potential registration errors from respiratory and cardiac motion and the different positions of various body parts when PET/CT and biopsies are performed at different times is another possible drawback. In patients in whom biopsy is considered the next diagnostic step, studies should be performed within a short interval and, whenever possible, in the same position used for the interventional procedure. The potential role of respiratory and cardiac gating for improved coregistration also needs to be investigated in more depth.

These potential limitations could probably be minimized with real-time PET/CT guidance. A recent case report demonstrated the use of intraprocedural real-time ${ }^{18} \mathrm{~F}-\mathrm{FDG}$ PET/CT in the biopsy of bone metastases from esophageal cancer (19). The technical feasibility, clinical success, and complication rates of PET/CT-guided biopsies were evaluated in a series of 126 patients that included 50 with lung masses (20). The target lesion and the biopsy needle location were reconfirmed using dedicated real-time PET/CT. The authors reported a success rate of $93 \%$ in obtaining representative tissue samples and a complication rate of $14 \%$ and concluded that biopsy guided by real-time PET/ CT data seems to be feasible and may optimize interventional procedures. Moreover, PET-positive lesions with no clear CT correlate may be accessible to tissue sampling using PET/CT information (20).

The routine clinical use of real-time PET/CT-guided FNA is currently limited, in part, by the potentially long machine time and the potential that personnel will be exposed to substantial ionizing radiation because of its persistence in the patient for several hours after radiopharmaceutical injection. The development of PET scanners with higher sensitivity could shorten scan times, thus increasing scanner availability, and also reduce the amount of radioactivity that needs to be administered. These limitations do not necessarily apply in the method proposed in the current study, since the diagnostic PET/CT acquisition is registered with the FNA CT. In an optimal setting, all that would be required is a suitable workstation and easy-to-use software in the procedure room.

\section{CONCLUSION}

The present study supports the concept that anatomic-metabolic guidance of FNA (using information provided by both ${ }^{18} \mathrm{~F}-\mathrm{FDG}$ PET and CT) may improve the accuracy of tissue sampling, decrease the incidence of FN results, and thus increase the probability of achieving a definitive diagnosis. Future work should focus on defining the technique and protocol to enable the clinical application of PET/CT to image-guided biopsy.

\section{DISCLOSURE}

The costs of publication of this article were defrayed in part by the payment of page charges. Therefore, and solely to indicate this fact, this article is hereby marked "advertisement" in accordance with 18 USC section 1734 . No potential conflict of interest relevant to this article was reported.

\section{REFERENCES}

1. Ohno Y, Hatabu H, Takenaka D, et al. CT-guided transthoracic needle aspiration biopsy of small $(<$ or $=20 \mathrm{~mm})$ solitary pulmonary nodules. AJR. 2003;180: 1665-1669.

2. Zhuang YP, Wang HY, Zhang J, et al. Diagnostic accuracy and safety of CTguided fine needle aspiration biopsy in cavitary pulmonary lesions. Eur J Radiol. 2013;82:182-186.

3. Pauwels EK, Ribeiro MJ, Stoot JH, et al. FDG accumulation and tumor biology. Nucl Med Biol. 1998;25:317-322.

4. Bomanji JB, Costa DC, Ell PJ. Clinical role of positron emission tomography in oncology. Lancet Oncol. 2001;2:157-164.

5. Hustinx R, Benard F, Alavi A. Whole-body FDG-PET imaging in the management of patients with cancer. Semin Nucl Med. 2002;32:35-46. 
6. Kubota R, Kubota K, Yamada S, et al. Microautoradiographic study for the differentiation of intratumoral macrophages, granulation tissues and cancer cells by the dynamics of fluorine-18-fluorodeoxyglucose uptake. J Nucl Med. 1994; 35:104-112.

7. Shreve PD, Anzai Y, Wahl RL. Pitfalls in oncologic diagnosis with FDG PET imaging: physiologic and benign variants. Radiographics. 1999;19:61-77.

8. Yap JT, Carney JP, Hall NC, et al. Image-guided cancer therapy using PET/CT. Cancer J. 2004;10:221-233.

9. Purandare NC, Kulkarni AV, Kulkarni SS, et al. ${ }^{18}$ F-FDG PET/CT-directed biopsy: does it offer incremental benefit? Nucl Med Commun. 2013;34:203-210.

10. Yokoyama K, Ikeda O, Kawanaka K, et al. Comparison of CT-guided percutaneous biopsy with and without registration of prior PET/CT images to diagnose mediastinal tumors. Cardiovasc Intervent Radiol. 2014;37:1306-1311.

11. Soret M, Bacharach SL, Buvat I. Partial-volume effect in PET tumor imaging. $J$ Nucl Med. 2007;48:932-945.

12. Kwee TC, Cheng G, Lam MG, et al. SUVmax of 2.5 should not be embraced as a magic threshold for separating benign from malignant lesions. Eur J Nucl Med Mol Imaging. 2013;40:1475-1477.

13. Pezeshk P, Sadow CA, Winalski CS, et al. Usefulness of ${ }^{18}$ F-FDG PET-directed skeletal biopsy for metastatic neoplasm. Acad Radiol. 2006;13:1011-1015.
14. Werner MK, Aschoff P, Reimold M, et al. FDG-PET/CT-guided biopsy of bone metastases sets a new course in patient management after extensive imaging and multiple futile biopsies. Br J Radiol. 2011;84:e65-e67.

15. Kim JM, Ryu JS, Kim TY, et al. ${ }^{18} \mathrm{~F}$-fluorodeoxyglucose positron emission tomography does not predict malignancy in thyroid nodules cytologically diagnosed as follicular neoplasm. J Clin Endocrinol Metab. 2007;92:1630-1634.

16. Kagna O, Solomonov A, Keidar Z, et al. The value of FDG-PET/CT in assessing single pulmonary nodules in patients at high risk of lung cancer. Eur J Nucl Med Mol Imaging. 2009;36:997-1004.

17. Shreve PD, Huy Bui CD. Artifacts and normal variants in FDG PET. In: Wahl RL, ed. Principles and Practice of PET and PET/CT. 2nd ed. Philadelphia, PA: Lippincott Williams \& Wilcott; 2009:139-168.

18. Tatli S, Gerbaudo VH, Mamede M, et al. Abdominal masses sampled at PET/CTguided percutaneous biopsy: initial experience with registration of prior PET/CT images. Radiology. 2010;256:305-311.

19. Win AZ, Aparici CM. Real-time FDG PET/CT-guided bone biopsy in a patient with two primary malignancies. Eur J Nucl Med Mol Imaging. 2013;40:1787-1788.

20. Cerci JJ, Pereira Neto CC, Krauzer C, et al. The impact of coaxial core biopsy guided by FDG PET/CT in oncological patients. Eur J Nucl Med Mol Imaging. 2013;40:98-103 